\title{
Analysis Bending Solutions of Clamped Rectangular Thick Plate
}

\author{
Yang Zhong and Qian Xu \\ School of Civil \& Hydraulic Engineering, Dalian University of Technology, Dalian, China \\ Correspondence should be addressed to Yang Zhong; zhongy@dlut.edu.cn
}

Received 11 January 2017; Accepted 11 April 2017; Published 24 May 2017

Academic Editor: Xiao-Qiao He

Copyright (C) 2017 Yang Zhong and Qian Xu. This is an open access article distributed under the Creative Commons Attribution License, which permits unrestricted use, distribution, and reproduction in any medium, provided the original work is properly cited.

\begin{abstract}
The bending solutions of rectangular thick plate with all edges clamped and supported were investigated in this study. The basic governing equations used for analysis are based on Mindlin's higher-order shear deformation plate theory. Using a new function, the three coupled governing equations have been modified to independent partial differential equations that can be solved separately. These equations are coded in terms of deflection of the plate and the mentioned functions. By solving these decoupled equations, the analytic solutions of rectangular thick plate with all edges clamped and supported have been derived. The proposed method eliminates the complicated derivation for calculating coefficients and addresses the solution to problems directly. Moreover, numerical comparison shows the correctness and accuracy of the results.
\end{abstract}

\section{Introduction}

The bending problem of rectangular thick plates with various combinations of boundary conditions is sparingly common in many engineering fields, such as aerospace, concrete pavements, and mechanical and structural engineering. Moreover with the development of modern industry, relatively more accurate and practical studies on bending plate are required. Problems involving rectangular plates fall into three distinct categories [1]: (a) plates with all edges simply supported; (b) plates with a pair of opposite edges simply supported; (c) plates which do not fall into any of the above categories.

The classical plate theory (CPT) is frequently used to analyze thin plates. This theory works on the assumption to ignore the transverse shear deformation and assumes that the normal to the middle plane before deformation remains straight and normal to the middle surface after deformation. Therefore, utilizing classical plate theory to analyze thick plates leads to somehow inaccurate and even wrong results.

Following classical plate theory, a series of theories have been developed by many researchers to analyze thick plates by taking account of the shear deformation, such as Mindlin's first-order, Reddy's third-order, and Reissner's higher-order shear deformation plate theory.
The couples governing differential equations of higher order could be obtained through the analogue theory mentioned above such as Mindlin's first-order, Reddy's thirdorder, and Reissner's higher-order shear deformation plate theory which have two more unknowns' variables in comparison with the classical plate theory. The following three types of methods can be used to solve the governing equation which are numerical methods including finite element method [2], Ritz energy method [3], and superposition method [4] and semianalytical methods which include Levy method [5], Navier method $[5,6]$, and the exact analytical methods which including symplectic geometry method $[7,8]$, and integral transform method [9]. The imposition of boundary conditions on the governing equations increased the mathematical complexity of the solution procedure. Therefore, the analytic bending solutions of rectangular thick plates are hard to solve. Furthermore, numerical methods could be used to solve the bending problems of plate. However, only the analytical method can give the exact solution, which is used to verify the results obtained from various numerical methods.

There are two common ways to deal with the plate problem. First way is to find new plate theories [10-15] which can reduce the number of unknown equations. Houari et al. [10] use the new simple higher-order shear deformation theory 
to analyze bending and free vibration of functionally graded plates. Tounsi et al. [11] perform the new 3-unknown nonpolynomial shear deformation theory for the buckling and vibration analyses of functionally graded material (FGM) sandwich plates. The abovementioned theories only dealt with three unknowns as the classical plate theory. Similarly, Beldjelili et al. [12] employ a four-variable refined plate theory to discuss the hygrothermomechanical bending behavior of sigmoid functionally graded material (S-FGM) plate resting on variable two-parameter elastic foundations. The second way is to find method to simplify the coupled governing differential equations of high order. For this purpose, decoupling method is used to handle such kind of problem.

In this context, the study focused on the improvement of modified Navier method to solve bending problem of rectangular plates with all edges clamped and supported. By using decoupling, modified Navier method has been modified into a new simple approach to solve the partial differential equations for Mindlin plate. In Section 2.1, the multiple differential equations have been decoupled while adding a new variable and ascending the equations' order. Two of the obtained equations are independent which can be solved directly, and another two equations are also much simpler than the already developed equations. In Section 2.2, generalized displacement variables in governing equations are obtained by using independent equations in the modified Navier method, and other solutions of the problem, namely, bending moments, have been obtained through related expressions of variables. In the end numerical comparison studies are shown to verify the results.

\section{Solution for Rectangular Thick Plate}

2.1. Decouple Mindlin Equations. The governing equations for bending problem of rectangular thick plates are given by

$$
\begin{aligned}
& \nabla^{2} w-\left(\frac{\partial \varphi_{x}}{\partial x}+\frac{\partial \varphi_{y}}{\partial y}\right)+\frac{q}{C}=0 \\
& \frac{\partial^{2} \varphi_{x}}{\partial x^{2}}+\frac{1-\mu}{2} \frac{\partial^{2} \varphi_{x}}{\partial y^{2}}+\frac{1+\mu}{2} \frac{\partial^{2} \varphi_{y}}{\partial x \partial y}+\frac{C}{D}\left(\frac{\partial w}{\partial x}-\varphi_{x}\right) \\
& =0 \\
& \frac{\partial^{2} \varphi_{y}}{\partial y^{2}}+\frac{1-\mu}{2} \frac{\partial^{2} \varphi_{y}}{\partial x^{2}}+\frac{1+\mu}{2} \frac{\partial^{2} \varphi_{x}}{\partial x \partial y}+\frac{C}{D}\left(\frac{\partial w}{\partial y}-\varphi_{y}\right) \\
& =0,
\end{aligned}
$$

where $\nabla^{2}=\partial^{2} / \partial x^{2}+\partial^{2} / \partial y^{2}, D$ is the flexural rigidity of the plate and its expression is $D=E h^{3} / 12\left(1-\mu^{2}\right), C$ is the shearing stiffness of the plate and its expression is $C=5 E h / 12(1+$ $\mu)$, and $E, \mu$, and $h$ are the elastic module, Poisson's ratio and the thickness of the plate, respectively. $w$ is the transverse deflection of the middle surface. $\varphi_{x}$ and $\varphi_{y}$ are the rotations of a normal line due to plate bending. $q$ is the load distribution function. The resultant bending moments, $M_{x}$ and $M_{y}$, the twisting moments $M_{x y}$ can be obtained; namely,

$$
\begin{aligned}
& M_{x}=-D\left(\frac{\partial \varphi_{x}}{\partial x}+\mu \frac{\partial \varphi_{y}}{\partial y}\right) \\
& M_{y}=-D\left(\frac{\partial \varphi_{y}}{\partial y}+\mu \frac{\partial \varphi_{x}}{\partial x}\right) \\
& M_{x y}=\frac{D(1-\mu)}{2}\left(\frac{\partial \varphi_{y}}{\partial y}+\frac{\partial \varphi_{x}}{\partial x}\right) .
\end{aligned}
$$

Another new variable $M$ is given for decoupling the governing equations (1)-(3). According to the left expression of (1), let

$$
M=\frac{\partial \varphi_{x}}{\partial x}+\frac{\partial \varphi_{y}}{\partial y}
$$

and (1) can be expressed as

$$
\nabla^{2} w-M+\frac{q}{C}=0
$$

Taking partial derivative of (2) and (3) with respect to $x$ and $y$, respectively, then considering (7), we can obtain

$$
\nabla^{2} M+\frac{C}{D}\left(\nabla^{2} w-M\right)=0 .
$$

Substituting (8) into (9), the independent differential equation about $M$ yield is obtained as follows:

$$
\nabla^{2} M-\frac{q}{D}=0
$$

Based on (8) and (10), the independent equation about $w$ is obtained as follows:

$$
\nabla^{4} w=\frac{q}{D}-\frac{\nabla^{2} q}{C}
$$

Taking partial derivative of (1) with respect to $x$ and $y$, respectively, is as follows:

$$
\begin{aligned}
& \nabla^{2} \frac{\partial w}{\partial x}-\left(\frac{\partial^{2} \varphi_{x}}{\partial x^{2}}+\frac{\partial^{2} \varphi_{y}}{\partial x \partial y}\right)+\frac{1}{C} \frac{\partial q}{\partial x}=0 \\
& \nabla^{2} \frac{\partial w}{\partial y}-\left(\frac{\partial^{2} \varphi_{y}}{\partial y^{2}}+\frac{\partial^{2} \varphi_{x}}{\partial x \partial y}\right)+\frac{1}{C} \frac{\partial q}{\partial y}=0 .
\end{aligned}
$$

Multiply (12) by coefficient $(1+\mu) / 2$, and then subtract with (2) to eliminate $\varphi_{y}$ in the equation. The expression between $w$ and $\varphi_{x}$ yields

$$
\nabla^{2} \varphi_{x}-Q \varphi_{x}=-Q \frac{\partial w}{\partial x}-\frac{1+\mu}{1-\mu} \frac{\partial}{\partial x}\left(\nabla^{2} w+\frac{q}{C}\right)
$$

The same as the derivation for (14), the expression between $w$ and $\varphi_{y}$ can be obtained as

$$
\nabla^{2} \varphi_{y}-Q \varphi_{y}=-Q \frac{\partial w}{\partial y}-\frac{1+\mu}{1-\mu} \frac{\partial}{\partial y}\left(\nabla^{2} w+\frac{q}{C}\right) .
$$


According to (8), further simplifying (14) and (15) yields

$$
\begin{aligned}
& \nabla^{2} \varphi_{x}-Q \varphi_{x}=-Q \frac{\partial w}{\partial x}-\frac{1+\mu}{1-\mu} \frac{\partial M}{\partial x} \\
& \nabla^{2} \varphi_{y}-Q \varphi_{y}=-Q \frac{\partial w}{\partial y}-\frac{1+\mu}{1-\mu} \frac{\partial M}{\partial y} .
\end{aligned}
$$

The basic governing equations are reexpressed as follows:

$$
\begin{aligned}
\nabla^{2} M-\frac{q}{D} & =0 \\
\nabla^{4} w & =\frac{q}{D}-\frac{\nabla^{2} q}{C} \\
\nabla^{2} \varphi_{x}-Q \varphi_{x} & =-Q \frac{\partial w}{\partial x}-\frac{1+\mu}{1-\mu} \frac{\partial M}{\partial x} \\
\nabla^{2} \varphi_{y}-Q \varphi_{y} & =-Q \frac{\partial w}{\partial y}-\frac{1+\mu}{1-\mu} \frac{\partial M}{\partial y} .
\end{aligned}
$$

2.2. Solution Method of Decoupled Equation. Considering the example of rectangular thick plates with all edges clamp supported, the solution of basic governing equations (1)-(3) is obtained through the modified Navier method. First, the boundary condition equations for CCCC plates are given by

$$
\begin{aligned}
x=0, a: & \\
w & =0 ; \\
\varphi_{x} & =0 ; \\
\varphi_{y} & =0 \\
y=0, b: \quad & \\
w & =0 ; \\
\varphi_{x} & =0 ; \\
\varphi_{y} & =0
\end{aligned}
$$

and (18) shows the basic form of boundary condition. The expressions of $w, \varphi_{x}$, and $\varphi_{y}$ are assumed as double sine series

$$
\begin{gathered}
w(x, y)=\sum_{n=1}^{\infty} \sum_{m=1}^{\infty} W_{n m} \sin \alpha_{n} x \sin \beta_{m} y \\
\varphi_{x}(x, y)=\sum_{n=1}^{\infty} \sum_{m=1}^{\infty} X_{n m} \sin \alpha_{n} x \sin \beta_{m} y \\
\varphi_{y}(x, y)=\sum_{n=1}^{\infty} \sum_{m=1}^{\infty} Y_{n m} \sin \alpha_{n} x \sin \beta_{m} y
\end{gathered}
$$

where $\alpha_{n}=n \pi / a$ and $\beta_{m}=m \pi / b$. Based on the definition of $M$ as (7), the boundary condition for $M$ is obtained as follows:

$$
\begin{aligned}
& x=0, a: M=0 \\
& y=0, b: M=0 .
\end{aligned}
$$

The expression of $M$ is also assumed as double sine series:

$$
M(x, y)=\sum_{n=1}^{\infty} \sum_{m=1}^{\infty} M_{n m} \sin \alpha_{n} x \sin \beta_{m} y .
$$

Expanding $q$ in the form of double sine series,

$$
q=\sum_{n=1}^{\infty} \sum_{m=1}^{\infty} Q_{n m} \sin \alpha_{n} x \sin \beta_{m} y,
$$

where $Q_{n m}$ is defined as

$$
Q_{n m}=\frac{4}{a b} \int_{0}^{a} \int_{0}^{b} q(x, y) \sin \alpha_{n} x \sin \beta_{m} y d x d y .
$$

Substituting (23) and (24) into (17a) gives

$$
\begin{gathered}
\sum_{n=1}^{\infty} \sum_{m=1}^{\infty} M_{n m}\left(\alpha_{n}{ }^{2}+\beta_{m}{ }^{2}\right) \sin \alpha_{n} x \sin \beta_{m} y \\
=-\sum_{n=1}^{\infty} \sum_{m=1}^{\infty} \frac{Q_{n m}}{D} \sin \alpha_{n} x \sin \beta_{m} y .
\end{gathered}
$$

According to the uniqueness theorem of Fourier expansion, equating the coefficient $M_{n m}$ in (26),

$$
\begin{gathered}
M_{n m}=-\frac{Q_{n m}}{D\left(\alpha_{n}{ }^{2}+\beta_{m}{ }^{2}\right)}=-\frac{4}{a b D\left(\alpha_{n}{ }^{2}+\beta_{m}{ }^{2}\right)} \\
\cdot \int_{0}^{a} \int_{0}^{b} q(x, y) \sin \alpha_{n} x \sin \beta_{m} y d x d y .
\end{gathered}
$$

Substituting (27) into (23), the expression for $M$ is obtained as follows:

$$
\begin{aligned}
& M(x, y)=-\frac{4}{a b D} \sum_{n=1}^{\infty} \sum_{m=1}^{\infty}\left[\frac{1}{\left(\alpha_{n}{ }^{2}+\beta_{m}{ }^{2}\right)}\right. \\
& \left.\quad \cdot \int_{0}^{a} \int_{0}^{b} q(x, y) \sin \alpha_{n} x \sin \beta_{m} y d x d y\right] \sin \alpha_{n} x \\
& \cdot \sin \beta_{m} y .
\end{aligned}
$$

Substituting (19) and (24) into (17b) yields the following result:

$$
\begin{aligned}
\sum_{n=1}^{\infty} \sum_{m=1}^{\infty} W_{n m}\left(\alpha_{n}{ }^{4}+\beta_{m}{ }^{4}+2 \alpha_{n}{ }^{2} \beta_{m}{ }^{2}\right) \sin \alpha_{n} x \sin \beta_{m} y \\
\quad=\sum_{n=1}^{\infty} \sum_{m=1}^{\infty}\left(\frac{1}{D}+\frac{\alpha_{n}{ }^{2}+\beta_{m}{ }^{2}}{C}\right) Q_{n m} \sin \alpha_{n} x \sin \beta_{m} y .
\end{aligned}
$$


According to the uniqueness theorem of Fourier expansion, $W_{m n}$ is obtained as

$$
\begin{aligned}
& W_{n m}=\left(\frac{1}{D\left(\alpha_{n}{ }^{2}+\beta_{m}{ }^{2}\right)}+\frac{1}{C}\right) \\
& \cdot\left(\frac{4}{a b} \int_{0}^{a} \int_{0}^{b} q(x, y) \sin \alpha_{n} x \sin \beta_{m} y d x d y\right) \\
& \cdot\left(\alpha_{n}{ }^{2}+\beta_{m}{ }^{2}\right)^{-1} .
\end{aligned}
$$

Substituting (30) into (19), the expression of $w$ is obtained as follows:

$$
\begin{aligned}
& w(x, y)=\sum_{n=1}^{\infty} \sum_{m=1}^{\infty} \frac{1}{\alpha_{n}^{2}+\beta_{m}^{2}}\left(\frac{1}{D\left(\alpha_{n}{ }^{2}+\beta_{m}^{2}\right)}+\frac{1}{C}\right) \\
& \cdot\left(\frac{4}{a b} \int_{0}^{a} \int_{0}^{b} q(x, y) \sin \alpha_{n} x \sin \beta_{m} y d x d y\right) \\
& \cdot \sin \alpha_{n} x \sin \beta_{m} y .
\end{aligned}
$$

And substituting (29) and (31) into (17c) and (17d) yielded

$$
\begin{aligned}
& \sum_{n=1}^{\infty} \sum_{m=1}^{\infty} X_{n m}\left(\alpha_{n}{ }^{2}+\beta_{m}{ }^{2}+Q\right) \sin \alpha_{n} x \sin \beta_{m} y \\
& =\sum_{n=1}^{\infty} \sum_{m=1}^{\infty}\left[M_{n m} \frac{1+\mu}{1-\mu}+W_{n m} Q\right] \alpha_{n} \cos \alpha_{n} x \sin \beta_{m} y \\
& \sum_{n=1}^{\infty} \sum_{m=1}^{\infty} Y_{n m}\left(\alpha_{n}{ }^{2}+\beta_{m}^{2}+Q\right) \sin \alpha_{n} x \sin \beta_{m} y \\
& =\sum_{n=1}^{\infty} \sum_{m=1}^{\infty}\left[M_{n m} \frac{1+\mu}{1-\mu}+W_{n m} Q\right] \beta_{m} \sin \alpha_{n} x \cos \beta_{m} y .
\end{aligned}
$$

Thus $M_{n m}$ and $W_{n m}$ obtained are shown as (27) and (30). Equating the unknown coefficients $X_{n m}$ and $Y_{n m}$ in (32), the expressions of $\varphi_{x}$ and $\varphi_{y}$ will be yielded as well. First unify the series core in a manner as follows:

$$
\begin{aligned}
& \cos \alpha_{n} x=\sum_{r=1}^{\infty} h_{r n} \sin \alpha_{r} x ; \quad 0<x<a \\
& \cos \beta_{m} y=\sum_{s=1}^{\infty} h_{s m} \sin \beta_{s} y ; \quad 0<y<b,
\end{aligned}
$$

where

$$
\begin{aligned}
& h_{r n}= \begin{cases}\frac{4 r}{\pi\left(r^{2}-n^{2}\right)} & n+r=\text { odd }, n-r=\text { odd } \\
0 & n+r=\text { even, } n-r=\text { even }\end{cases} \\
& h_{s m}= \begin{cases}\frac{4 s}{\pi\left(s^{2}-m^{2}\right)} & s+m=\text { odd, } m-s=\text { odd } \\
0 & m-s=\text { even, } m-s=\text { even }\end{cases}
\end{aligned}
$$

based on (33), expand $\cos \alpha_{n} x$ and $\cos \beta_{m} y$ in the form of $\sin$ Fourier series, and reset the dummy variables:

$$
\begin{aligned}
& \sum_{n=1}^{\infty} \sum_{m=1}^{\infty} \sin \alpha_{n} x \sin \beta_{m} y X_{n m}\left(\alpha_{n}{ }^{2}+\beta_{m}{ }^{2}+Q\right) \\
& =\sum_{m=1}^{\infty} \sum_{n=1}^{\infty} \sin \alpha_{n} x \sin \beta_{m} y \sum_{r=1}^{\infty}\left[M_{r m} \frac{1+\mu}{1-\mu}+W_{r m} Q\right] \\
& \cdot h_{n r} \alpha_{r} \\
& \sum_{n=1}^{\infty} \sum_{m=1}^{\infty} \sin \alpha_{n} x \sin \beta_{m} y Y_{n m}\left(\alpha_{n}{ }^{2}+\beta_{m}{ }^{2}+Q\right) \\
& =\sum_{n=1}^{\infty} \sum_{m=1}^{\infty} \sin \alpha_{n} x \sin \beta_{m} y \\
& \quad \cdot \sum_{s=1}^{\infty}\left[M_{n s} \frac{1+\mu}{1-\mu}+W_{n s} Q\right] h_{m s} \beta_{s} .
\end{aligned}
$$

Finally, equating the coefficients in (35) directly according to the uniqueness theorem of Fourier expansion,

$$
\begin{aligned}
& X_{n m}=-\left(\alpha_{n}{ }^{2}+\beta_{m}{ }^{2}+Q\right)^{-1} \sum_{r=1}^{\infty}\left[M_{r m} \frac{1+\mu}{1-\mu}+W_{r m} Q\right] \\
& \cdot h_{n r} \alpha_{r} \\
& Y_{n m}=-\left(\alpha_{n}{ }^{2}+\beta_{m}{ }^{2}+Q\right)^{-1} \sum_{s=1}^{\infty}\left[M_{n s} \frac{1+\mu}{1-\mu}+W_{n s} Q\right] \\
& \cdot h_{m s} \beta_{s} .
\end{aligned}
$$

Substituting (36) and (37) into (20) and (21), respectively, the expressions of $\varphi_{x}$ and $\varphi_{y}$ are obtained as follows:

$$
\begin{aligned}
& \varphi_{x}(x, y)=\sum_{n=1}^{\infty} \sum_{m=1}^{\infty} \frac{1}{\alpha_{n}{ }^{2}+\beta_{m}{ }^{2}+Q} \sum_{r=1}^{\infty} \frac{Q_{r m}}{\alpha_{r}{ }^{2}+\beta_{m}{ }^{2}}\left\{\frac{1+\mu}{D(1-\mu)}+\left[\frac{1}{D\left(\alpha_{r}{ }^{2}+\beta_{m}{ }^{2}\right)}+\frac{1}{C}\right] Q\right\} h_{n r} \alpha_{r} \sin \alpha_{n} x \sin \beta_{m} y \\
& \varphi_{y}(x, y)=\sum_{n=1}^{\infty} \sum_{m=1}^{\infty} \frac{1}{\alpha_{n}{ }^{2}+\beta_{m}{ }^{2}+Q} \sum_{s=1}^{\infty} \frac{Q_{n s}}{\alpha_{n}{ }^{2}+\beta_{s}{ }^{2}}\left\{\frac{1+\mu}{D(1-\mu)}+\left[\frac{1}{D\left(\alpha_{n}{ }^{2}+\beta_{s}{ }^{2}\right)}+\frac{1}{C}\right] Q\right\} h_{m s} \beta_{s} \sin \alpha_{n} x \sin \beta_{m} y .
\end{aligned}
$$


TABLE 1: Nondimensional deflection and moment of a square plate, under uniform pressure and clamped (CCCC) boundary conditions.

\begin{tabular}{cccc}
\hline$a / b \quad m, n$ & $\left.w\right|_{x=a / 2, y=b / 2}\left(q a^{4} \cdot D^{-1}\right)$ & $\left.M_{x}\right|_{x=0, y=b / 2}\left(q a^{2}\right)$ \\
\hline \multirow{4}{*}{$3, n=10$} & -0.003615 & -0.04225 \\
& $m, n=15$ & -0.003610 & -0.04295 \\
& $m, n=20$ & -0.003611 & -0.04353 \\
& FEM results & -0.00361 & -0.0435 \\
\hline & $m, n=10$ & -0.002116 & -0.04008 \\
5 & $m=15$ & -0.002114 & -0.04321 \\
& $m, n=20$ & -0.002114 & -0.04589 \\
& FEM results & -0.00211 & -0.0458 \\
\hline & $m, n=10$ & -0.001483 & -0.0432 \\
& $m, n=15$ & -0.001483 & -0.04739 \\
& $m, n=20$ & -0.001483 & -0.05140 \\
& FEM results & -0.00148 & -0.0510 \\
\hline
\end{tabular}

Similarly substituting (38) and (39) into (4) and (5), the expressions of $M_{x}$ and $M_{y}$ can be obtained as follows:

$$
\begin{aligned}
M_{x} & =-D \sum_{n=1}^{\infty} \sum_{m=1}^{\infty}\left\{\alpha_{n} \cos \alpha_{n} x \sin \beta_{m} y\left(\alpha_{n}{ }^{2}+\beta_{m}{ }^{2}+Q\right)^{-1}\right. \\
& \cdot \sum_{r=1}^{\infty}\left[-\frac{Q_{r m}}{D\left(\alpha_{r}{ }^{2}+\beta_{m}{ }^{2}\right)} \frac{1+\mu}{1-\mu}+Q\left(\frac{Q_{r m}}{D}+\frac{\alpha_{r}{ }^{2}+\beta_{m}{ }^{2}}{C}\right)\left(\alpha_{r}{ }^{4}+\beta_{m}{ }^{4}+2 \alpha_{r}{ }^{2} \beta_{m}{ }^{2}\right)^{-1}\right] h_{n r} \alpha_{r}+\mu \beta_{m} \sin \alpha_{n} x \cos \beta_{m} y \\
& \left.\cdot\left(\alpha_{n}{ }^{2}+\beta_{m}{ }^{2}+Q\right)^{-1} \sum_{s=1}^{\infty}\left[-\frac{Q_{n s}}{D\left(\alpha_{n}{ }^{2}+\beta_{s}{ }^{2}\right)} \frac{1+\mu}{1-\mu}+Q\left(\frac{Q_{n s}}{D}+\frac{\alpha_{n}{ }^{2}+\beta_{s}{ }^{2}}{C}\right)\left(\alpha_{n}{ }^{4}+\beta_{s}{ }^{4}+2 \alpha_{n}{ }^{2} \beta_{s}{ }^{2}\right)^{-1}\right] h_{m s} \beta_{s}\right\} \\
M_{y} & =-D \sum_{n=1}^{\infty} \sum_{m=1}^{\infty}\left\{\beta_{m} \sin \alpha_{n} x \cos \beta_{m} y\left(\alpha_{n}{ }^{2}+\beta_{m}{ }^{2}+Q\right)^{-1}\right. \\
& \cdot \sum_{s=1}^{\infty}\left[-\frac{Q_{n s}}{D\left(\alpha_{n}{ }^{2}+\beta_{s}{ }^{2}\right)} \frac{1+\mu}{1-\mu}+Q\left(\frac{Q_{n s}}{D}+\frac{\alpha_{n}{ }^{2}+\beta_{s}{ }^{2}}{C}\right)\left(\alpha_{n}{ }^{4}+\beta_{s}{ }^{4}+2 \alpha_{n}{ }^{2} \beta_{s}{ }^{2}\right)^{-1}\right] h_{m s} \beta_{s}+\mu \alpha_{n} \cos \alpha_{n} x \sin \beta_{m} y \\
& \left.\cdot\left(\alpha_{n}{ }^{2}+\beta_{m}{ }^{2}+Q\right)^{-1} \sum_{r=1}^{\infty}\left[-\frac{Q_{r m}}{D\left(\alpha_{r}{ }^{2}+\beta_{m}{ }^{2}\right)} \frac{1+\mu}{1-\mu}+Q\left(\frac{Q_{r m}}{D}+\frac{\alpha_{r}{ }^{2}+\beta_{m}{ }^{2}}{C}\right)\left(\alpha_{r}{ }^{4}+\beta_{m}{ }^{4}+2 \alpha_{r}{ }^{2} \beta_{m}{ }^{2}\right)^{-1}\right] h_{n r} \alpha_{r}\right\} .
\end{aligned}
$$

\section{Numerical Example}

A thick plate with all edges clamped (CCCC) has been taken as a numerical example to justify the correctness of the above solution. The length and width of the plate are $a=b$, with the Poisson ratio of $\mu=0.3$. Figure 1 shows the change in deflection of the plate. Table 1 highlights the comparison of nondimensional deflection results with solutions given by FEM, which shows that the results obtained are in accordance with the ones given before, and proves the correctness of the above method and the derivations.

\section{Conclusion}

The decoupling method and the modified Navier's solution have been used together in this study for a simple analysis of rectangular thick plates with all edges clamped and supported. Unlike the original modified Navier method, the proposed approach does not need complicated matrix derivations for calculating the coefficients. The procedure for solving the bending rectangular thick plates with all edges clamped is made simpler than before. Moreover the proposed method can be further extended to address the problem 




Figure 1: The deflections of a square plate.

of rectangular thick plates with other combinations of free and simply supported boundary conditions. The proposed method has many practical applications and can be used in foundation design of high-rise building and rigid pavements of highway and airport. Additionally, the plate support problems such as point supports and spring supports can be solved well analytically by utilizing similar approach, which would expectantly develop inspiring extensions in the field. Moreover, the results obtained from numerical example validate the precision and correctness of method and derivations.

\section{Conflicts of Interest}

The authors declare that they have no conflicts of interest.

\section{Acknowledgments}

The work described in this paper was supported by the National Natural Science Foundation of China no. 10782039.

\section{References}

[1] K. Bhaskar and B. Kaushik, "Simple and exact series solutions for flexure of orthotropic rectangular plates with any combination of clamped and simply supported edges," Composite Structures, vol. 63, no. 1, pp. 63-68, 2004.

[2] S. Weiming and Y. Guangsong, "Rational finite element method for elastic bending of reissner plates," Applied Mathematics and Mechanics, vol. 20, no. 2, pp. 193-199, 1999.

[3] A. W. Leissa and F. W. Niedenfuhr, "A study of the cantilevered square plate subjected to a uniform loading," Journal of the Aero/Space Sciences, vol. 29, no. 2, 1962.

[4] M. K. Huang and H. D. Conway, "Bending of a uniformly loaded rectangular plate with two adjacent edges clamped and the others either simply supported or free," Journal of Apply Mechanics, vol. 19, pp. 451-460, 1952.

[5] S. P. Timoshenko and S. Woinowsky-Krieger, Theory of Plates and Shells, McGraw-Hill, 1959.

[6] H. R. H. Kabir and R. A. Chaudhuri, "Boundary-continuous fourier solution for clamped Mindlin plates," Journal of Engineering Mechanics, vol. 118, no. 7, pp. 1457-1467, 1992.

[7] Y. Zhong and R. Li, "Exact bending analysis of fully clamped rectangular thin plates subjected to arbitrary loads by new symplectic approach," Mechanics Research Communications, vol. 36, no. 6, pp. 707-714, 2009.
[8] R. Li, B. Wang, and P. Li, "Hamiltonian system-based benchmark bending solutions of rectangular thin plates with a corner point-supported," International Journal of Mechanical Sciences, vol. 85, pp. 212-218, 2014.

[9] Y. Zhong and J.-H. Yin, "Free vibration analysis of a plate on foundation with completely free boundary by finite integral transform method," Mechanics Research Communications, vol. 35, no. 4, pp. 268-275, 2008.

[10] M. S. Houari, A. Tounsi, A. Bessaim, and S. Mahmoud, "A new simple three-unknown sinusoidal shear deformation theory for functionally graded plates," Steel and Composite Structures, vol. 22, no. 2, pp. 257-276, 2016.

[11] A. Tounsi, M. S. Houari, and A. Bessaim, "A new 3-unknowns non-polynomial plate theory for buckling and vibration of functionally graded sandwich plate," Structural Engineering and Mechanics, vol. 60, no. 4, pp. 547-565, 2016.

[12] Y. Beldjelili, A. Tounsi, and S. Mahmoud, "Hygro-thermomechanical bending of S-FGM plates resting on variable elastic foundations using a four-variable trigonometric plate theory," Smart Structures and Systems, vol. 18, no. 4, pp. 755-786, 2016.

[13] H. Hebali, A. Tounsi, M. S. A. Houari, A. Bessaim, and E. A. A. Bedia, "New quasi-3D hyperbolic shear deformation theory for the static and free vibration analysis of functionally graded plates," ASCE, Journal of Engineering Mechanics, vol. 140, no. 2, pp. 374-383, 2014.

[14] A. Hamidi, M. S. A. Houari, S. R. Mahmoud, and A. Tounsi, "A sinusoidal plate theory with 5-unknowns and stretching effect for thermomechanical bending of functionally graded sandwich plates," Steel and Composite Structures, vol. 18, no. 1, pp. 235-253, 2015.

[15] A. Mahi, E. A. Adda Bedia, and A. Tounsi, "A new hyperbolic shear deformation theory for bending and free vibration analysis of isotropic, functionally graded, sandwich and laminated composite plates," Applied Mathematical Modelling. Simulation and Computation for Engineering and Environmental Systems, vol. 39, no. 9, pp. 2489-2508, 2015. 


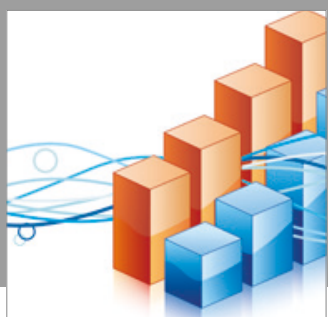

Advances in

Operations Research

vatersals

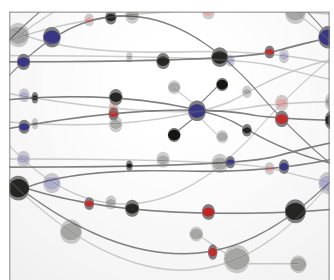

\section{The Scientific} World Journal
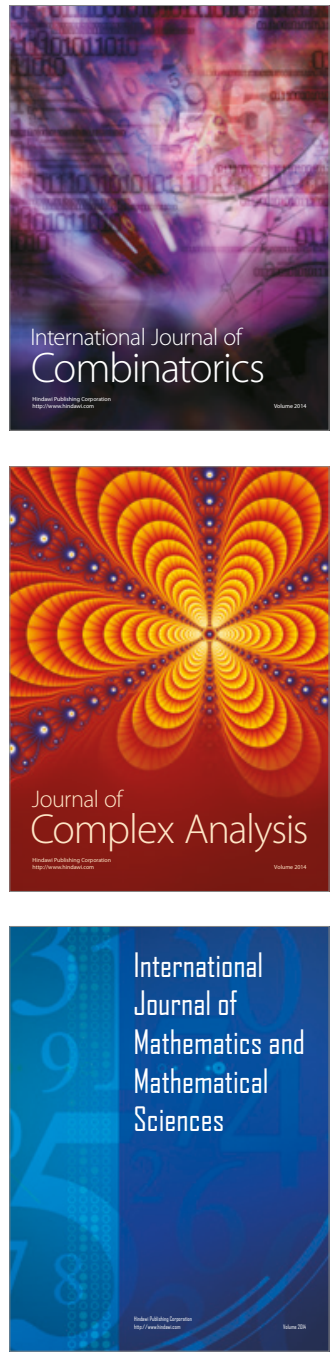
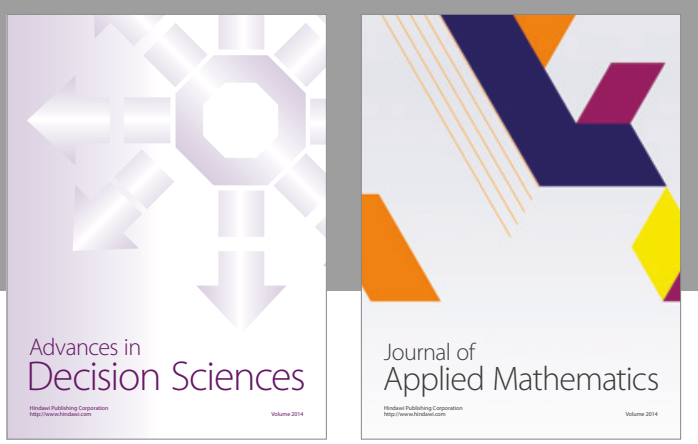

Algebra

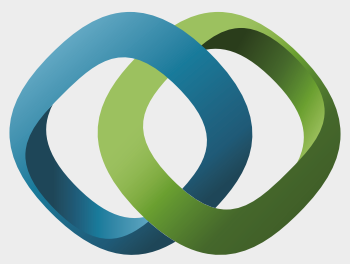

\section{Hindawi}

Submit your manuscripts at

https://www.hindawi.com
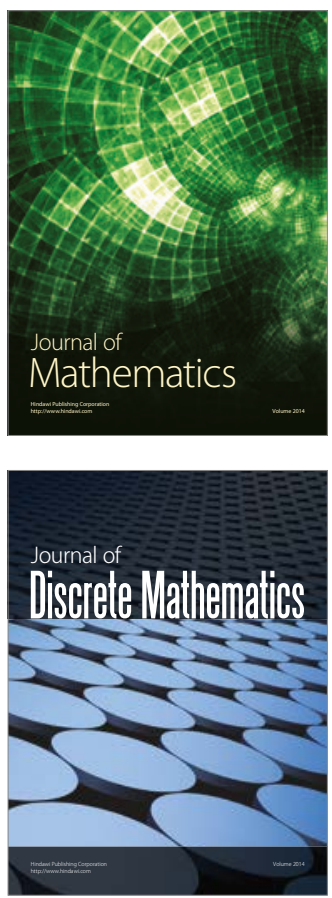

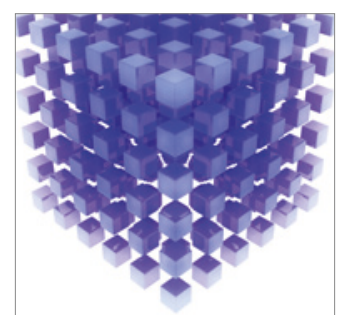

Mathematical Problems in Engineering
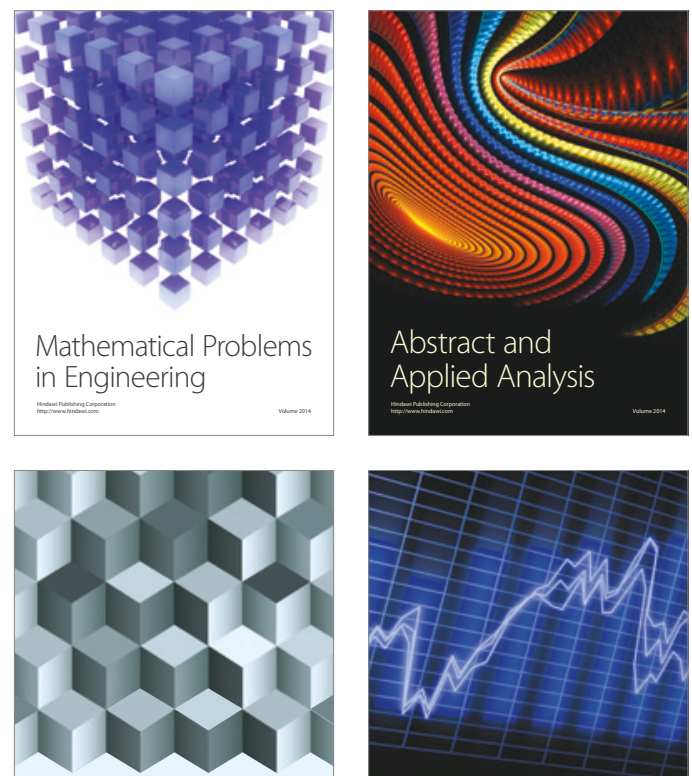

Journal of

Function Spaces

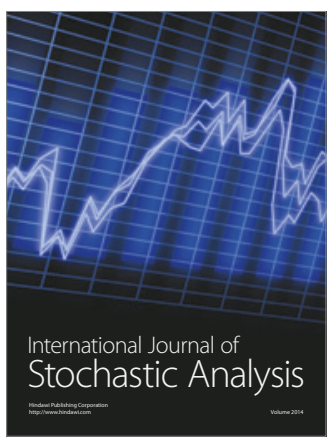

Probability and Statistics
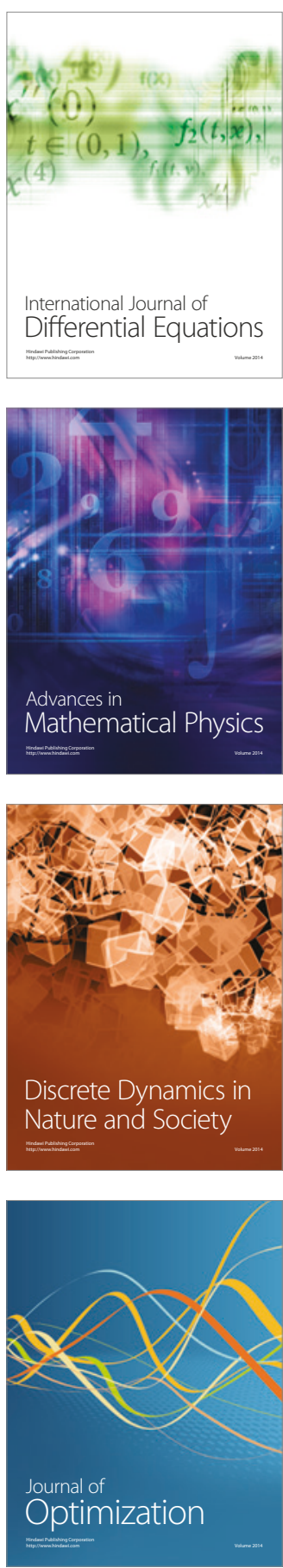